\title{
3D Digitizing Technology in Product Reverse Design
}

\author{
Qingjin Peng \\ Hector Sanchez \\ Department of Mechanical and Manufacturing Engineering \\ University of Manitoba \\ Winnipeg, Manitoba, R3T 5V6, Canada \\ pengq@cc.umanitoba.ca
}

\begin{abstract}
The reverse design develops new products based on the improvement of existing products. The shape recovery of three-dimensional (3D) objects is the basis of the product reverse design. $3 D$ digitization technology is an important tool for the $3 D$ shape recovery. This paper analyses the current $3 D$ data acquisition technology. The accuracy and performance of the $3 D$ laser scanner is evaluated. A cost-effective approach is proposed to recover $3 D$ shape of objects using a structured-light technique. Details of the proposed method are described. Application examples are presented. The accuracy is evaluated using a coordinate measuring machine.
\end{abstract}

\section{Introduction}

Reverse design (RD) is an improvement-type product design. One of RD's features is that the development of new products is based on the improvement of existing products. RD is widely used in various areas of product development. Examples of the RD applications include: rapid manufacturing of complex components [1]; extracting machining features and machining operations [2]; creating CAD models of the die and machining the die [3]; integrating with product rapid prototyping [4]; and Internet-based applications of product design [5].

The recovery of three-dimensional (3D) shape of an object is the basis of $\mathrm{RD}$. Coordinate measuring machines (CMMs) are traditional $\mathrm{RD}$ tools used in manufacturing industry. CMMs provide a high measuring accuracy and stability, but disadvantages are the contact measurement and a point-to-point slow measuring process. There is a number of factors that affect the digitizing uncertainty of CMMs, such as travel speeds of the probe, pitch values, probe angles, probe sizes, and part sizes.

The development of 3D digital technology in recent years has provided effective tools of the shape recovery for $\mathrm{RD}$. Besides CMMs, vision-based technology has significant applications in 3D shape recovery. Some examples include: an image-based RD to measure shape of sheet metal parts [6]; the vision sensor for free-form surfaces reconstruction [7]; combining stereo-vision and shape-from-shading recovery for obtaining reliable shape [8]; and scanning the surface of an unknown object through the utilization of optical range sensors [9].

The technology to capture 3D data of objects has been remarkable improved in recent years. Advanced software and increasingly powerful computers allow a large database and fast data post-processing. 3D scanners play an important role in vision-based 3D scanning technology. There has been much research to improve the measuring range and accuracy of the 3D canners. For instance, a framework was used for automatic planning of the laser scanning operation [10]; a laser scanning measurement technology was presented to acquire the $3 \mathrm{D}$ profile information of large objects [11]; and multiple patches was proposed in scanning of freeform-shaped models [12]. There are many commercial products available in market.

However, there is not a simple solution to select and use 3D scanning techniques because of the variable performance and high expense. Based on the research of the authors in 3D scanner applications and development, this paper first discusses and evaluates current $3 \mathrm{D}$ scanning technology, and then proposes a cost-effective prototyping of $3 \mathrm{D}$ digitizer in the product reverse design.

\section{3D digitization technology}

3D digitization technology can be classified as contact and non-contact techniques based on the method of 3D data acquisition as shown in Figure 1. The contact means that the measuring probe touches the recovery surface of objects during the data acquisition.

Contact data acquisition obtains data using a contact measuring process. The devices include joined arms and CMMs. A joined arm likes a robot that has a mechanical arm to follow the contour of the measured 


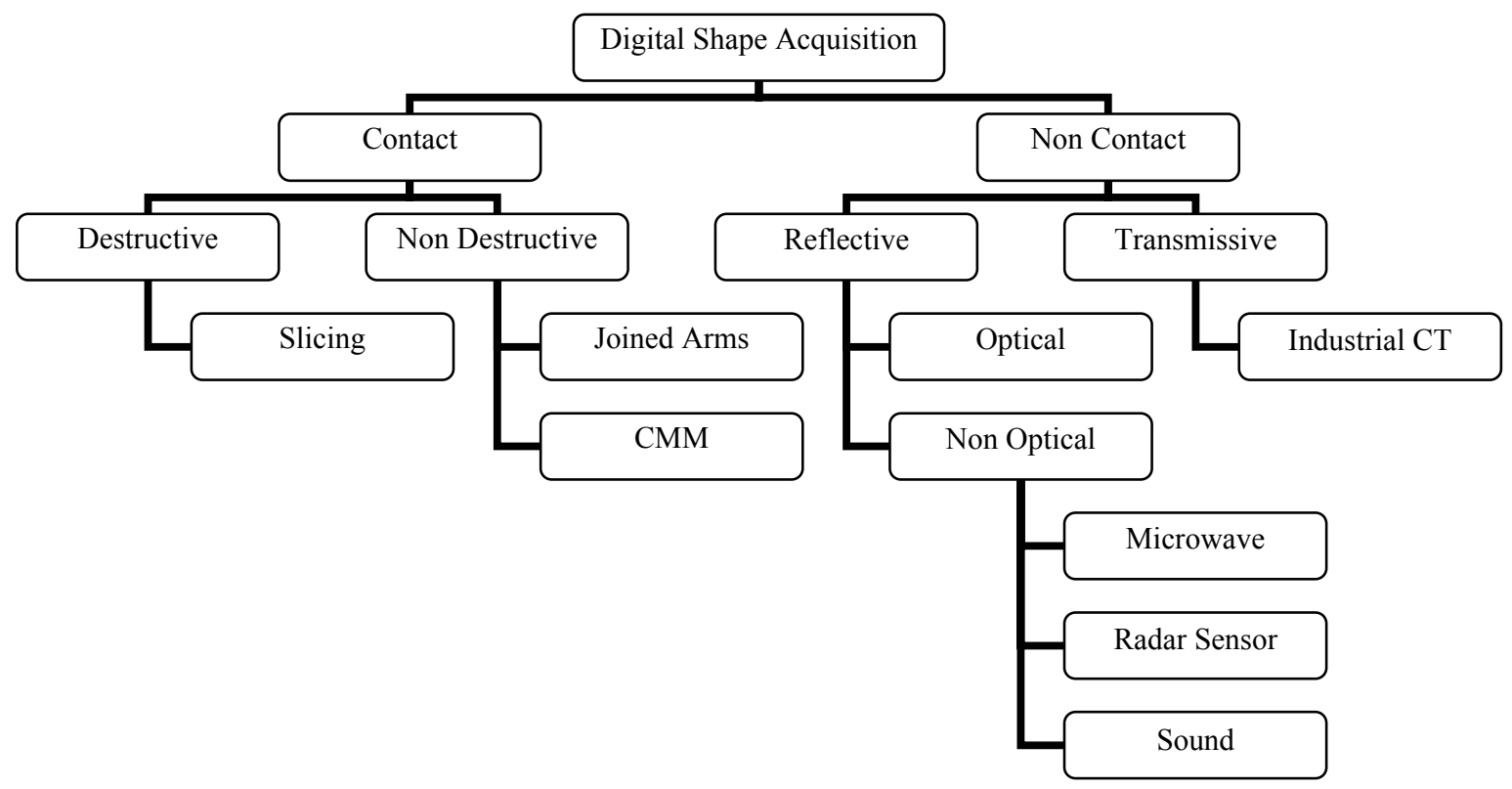

Figure 1. The classification of 3D digitization technology

object and send 3D data of the registered coordinates into the computer to form the 3D surface. The joined arm has disadvantages of the operation including timeconsuming and a high skilled labour required to move the arm from one point to another. CMMs are widely used in industry for dimensional inspection of manufactured parts. The most recent developments have led to the probe sensing edges and surfaces to be measured on the part. A position sensing system can report spatial coordinates of the probe at each measured location. These coordinate locations are then processed by measurement software to determine locations, dimensions, and geometry of the part. Despite the significant improvements in CMMs' performance over the past decade, several factors still limit CMMs' accuracy, speed, and economic utility. Thermal variations in the CMM environment can cause expansion and contraction of the individual component which distorts the kinematic structure, resulting in positioning errors of the probe tip. For this reason, virtually all CMMs used for moderate- to highprecision inspection are housed in specially constructed rooms with carefully controlled environments thereby substantially increasing the cost of the CMM installation and use.

Destructive and non-destructive methods are used in contact measuring process to obtain the surface which can be touched during the measurement of objects. The destructive technique gets the touched surface by cutting or slicing object into pieces. Non- destructive methods can only measure the profile of an object.

Non-Contact data acquisition technology uses an energy source, such as laser, white light, microwave, radar, and ultrasonic sound, to obtain 3D data of an object without touching the surface of objects in the measurement. There are two techniques used to receive signals of the energy source from measured surface: reflective and transmissive methods. Reflective method uses a receiver, such as a camera, to get the reflecting source (such as light) from the surface of objects. According to the source used, reflective devices are divided into optical devices and non-optical devices which use microwave, radar sensor or ultra sound. Non-optical systems are based on a time-delay measurement to calculate the distance traveled by sound or microwaves. Transmissive methods get a series of slices when the source is emitted through the part. These slices can be used to produce volumetric data or feature extraction. Examples of using this technique include ultrasound, magnetic resonance imaging (MRI), X-ray computed tomography (CT), and confocal microscopes. This research focuses on optical scanners

\section{3D reconstruction technologies of optical scanners}

A general process of optical scanner-based 3D shape reconstruction is shown in Figure 2. Point clouds 
are data acquired from objects scanned by a 3D optical scanner. A few scans may be needed to acquire different views of an object. The post-processing is the most important work to generate final structure of the object after scanning. It is a time-consuming process. The software makes triangles using data of point clouds. The data from each scan are constructed as triangle formations, and are merged to form a polygon mesh after deleting redundant data and filling holes of the surface recovered. Then, the software generates the feature curve and NURBS curve from polygon data. Finally, NURBS surfaces form a completed 3D shape of the object after rendering. The data can be saved as a standard data format for further applications in CAD/CAM systems.

There are different optical 3D scanners available in market based on technology used. The category of optical 3D scanners can be divided into the laser-based, white-light-based and radar-based scanners. The radarbased scanner has the ability to measure a large range of distance but with low accuracy. It cannot meet the requirement of manufacturing accuracy in product development. The measuring range and accuracy are both important factors for scanner applications in RD. Examples of applications may be a large-sized object such as a room or a car, or a small-sized object such as a machine component. Four scanners were initially analyzed by the authors for purchase purpose based on the performance and cost. They are Steibichler COMET 400 Optical Digitizing System, Minolta VIVID 900 non-contact 3D digitizer, 3D scanner from 3D Digital Corp., and ShapeGrabber's 3D scanner. Besides the measuring accuracy of the scanners, other performances, such as scanning speed, easy use, system reliability, and standard of data output format, were also evaluated. All of these scanners provide a similar accurate range (50-200um) in the test. ShapeGrabber scanner was selected finally to purchase based on the balance of the performances, supplied software and price. The scanner is shown in Figure 3. It uses different-sized scanning heads for different measurement ranges to achieve the required volume and accuracy.

\section{The evaluation and analysis of the 3D laser scanner}

Two objects were selected to evaluate the performance of ShapeGrabber's 3D laser scanner in order to understand the detail of its applications. The scanner's operation uses central software to control scanning process. The software can automatically detect the scanner head used on the apparatus and prompt users to use a certain calibration plate. Simply the small scanner head goes with the small calibration plate conversely the large plate goes with large scanner head. The calibration is required when the head changes, or after scanner is adjusted, or the scanner is not used for a long time. The scanner is calibrated by following instructions for the optimal height in which the plate should be located. The calibration data can be saved for next use. Then a configurator is used to set the scanning range, resolution, intensity of laser beam, and depth of measuring field. Now the scanner is ready to scan the object. A completed object is usually required a few scans to obtain its whole surface. A satellite dish and a wind generator blade were selected as test samples. The recovered shapes are shown in Figures 4 and 5.

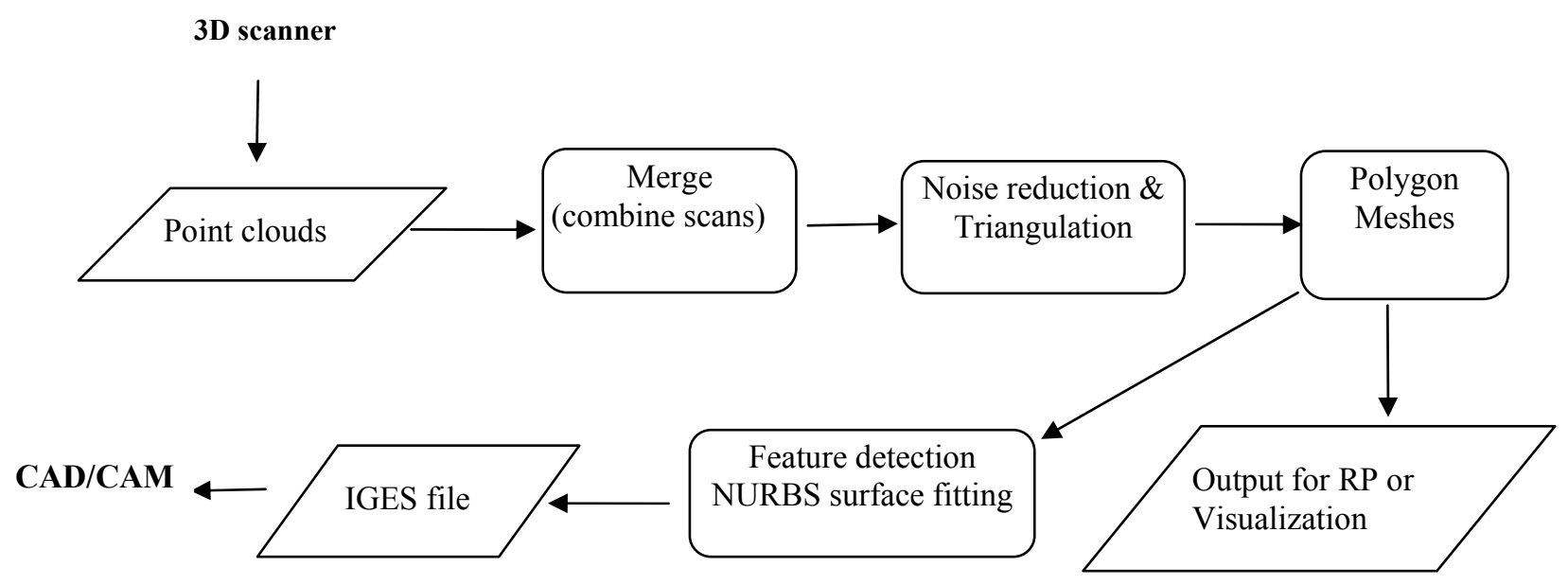

Figure 2. A general process of the optical scanner-based 3D shape reconstruction 


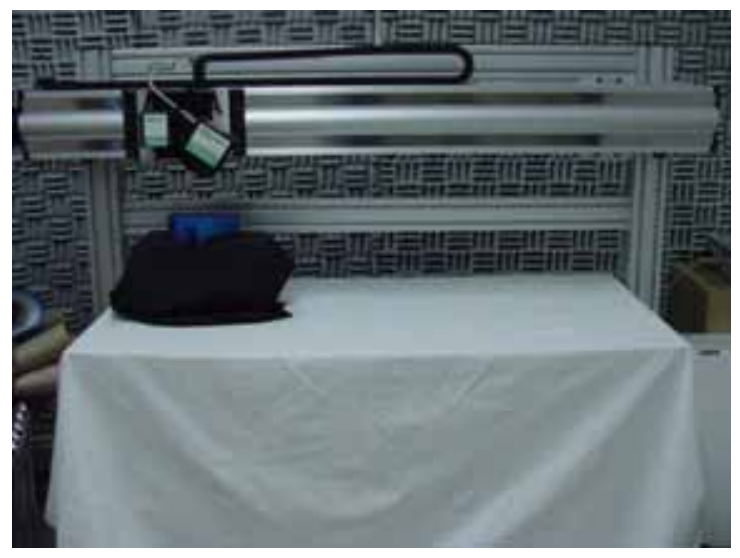

Figure 3. ShapeGrabber 3D laser scanner.

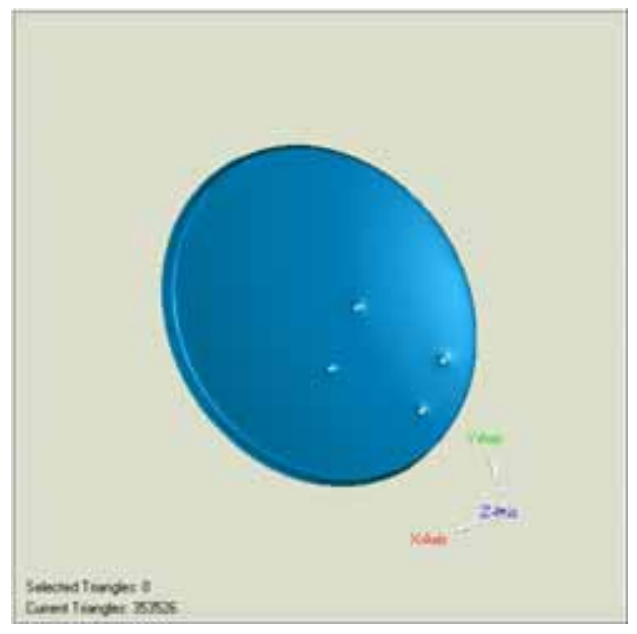

Figure 4. A satellite dish.

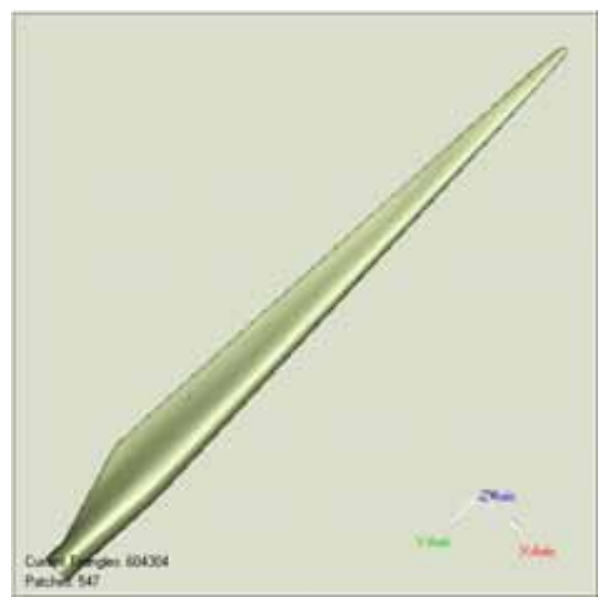

Figure 5. A wind generator blade.
The laser scanner is easier to operate than CMMs. It takes much less time to obtain the entire data. However, true industrial scanners service a much wider range of sizes and materials. Generally, an object requires multiple scans to obtain full data of the object. The acquired data from canning is a "cloud of points". Software must register one scan to another for the part completion. Therefore, software for data acquisition and processing is an important part of 3D digitization. The experience is required to arrange scanning process and number of scans to get an entire surface of the object. Especially, the post-processing is important to get the final recovered 3D shape. It usually forms an object shape using polygon/NURBS surfaces from scanned data. The effective algorithms will reduce the date size, shorten the processing time and form a complete object shape from the least scanning times. Generally, the basic functions required for the software of a scanner post-processing include follows:

- Ability to input multiple data formats to process.

- De-noising scanned data;

- Creation of polygonal models from cloud data;

- Boundary edge repair tools

- Hole filling

- Feature detection

- Creation of NURBS surface from polygon models

- Patch organization and merging tools

- Wide variety of 3D export formats.

Challenges in the data post-processing include as follows:

- Aligning point clouds together by picking out three common reference points: each scan must have enough common points to reference features that are on the previous and following scans.

- Control unmanageable combined point cloud: over-sized point cloud, such as more than 4 million points, may cause freezing or unacceptable rendering times.

- Control holes development: to reduce points with the same rate when point reducing is required. This causes holes to develop where spares clouds exist. The holes have to be relaxed and filled.

- Files exporting: the small-sized file is easier to be exported into CAD systems for further manipulation of the object. Balancing for small size with surface preservation is a tedious but vital work.

There has been much research to improve data processing ability of the software. The research includes the new algorithm that can improve surface reconstruction from a set of scattered 3D [13]; the suggestion to segment data into constituent surface 
patches, and to generate an accurate solid model [14]. There are also commercial software tools available to support the data post-processing, such as Polyworks and Geomegic software.

Geomegic studio was selected to find the need of data post-processing in this research. Geomagic Studio is a software tool for reverse engineering developed by Raindrop Geomagic, Inc. It has functions including the scan registration of multiple point clouds, point cloud noise reduction, filling holes of scanned data, polygon creation, data repair and editing, and NURBS surface creation.

Following solutions were generated from the shape recovery of two samples: the satellite dish and the wind generator blade:

- The use of laser scanner is much easier than CMMs operation to obtain cloud data,

- Post-processing takes more than $90 \%$ time in the object recovery,

- A complex shape will need multiple scans, which increases processing time,

- Fill missed parts or holes is time-consuming,

- Laser scanner cannot scan transparent objects, it is necessary to spray a film on the surface of the transparent object.

- Experience and skill are required in the processing.

- Exported data to $\mathrm{CAD}$ are not complete compatible.

A CMM is used to evaluate measuring accuracy of the laser scanner. The comparison of scanned data by the laser scanner and measured data by the CMM is shown in Fig 6. It is a top view of the part in the test. The profile, holes, and slot are features used to compare. The both data are very close from the view. It seems that the laser scanner can be used as a measuring tool in some areas of manufacturing to replace CMMs.

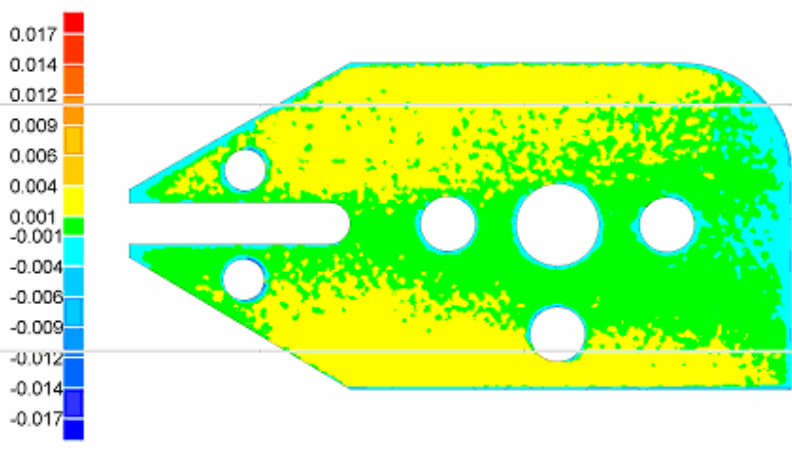

Figure 6. The accuracy comparison.

Besides laser scanners, white light-based scanners have the increasing applications in the field. These techniques intend to improve limitations of the laser scanners, including measuring range, accuracy, and cost. White light-based scanners are based on photometric stereo technique using two or more CCD cameras to passively capture the scene. The points on one image have to correspond to the points on other images in order to obtain the 3D information of an object.

The key of these techniques is the data processing efficiency of the algorithm. It is time-consuming that the computer software matches both images for the photometric stereo technique. It may not be able to do a real-time recovery processing. A referenced pattern may be projected to the surface of object to achieve a quick match processing. In summary, there is not a cost-effective solution for industrial applications with current 3D scanning techniques. The commercial 3D shape capturing products cost from $\$ 30,000$ to $\$ 100,000$. It is not affordable by most of small and medium sized industries. Following parts of the paper introduce the authors' research for a cost-effective solution of the 3D shape acquisition.

\section{The proposed 3D data acquisition method}

\subsection{The method introduction}

The proposed method uses a white light-based shape recovery technique. The additional signs are required to project onto the object surface to increase clue of the shape change in a smooth surface. This method is called the structured-light based technique. It uses a spatial distribution lighting source to project sheets or beams of light onto objects, which is then captured by camera(s) for the 3D shape acquisition [15]. There are different strategies to project lighting patterns onto objects to recover the 3D shape of objects [16]. The purpose of these strategies is to improve the accuracy of shape recovery and increase flexibility of techniques [17]. They do provide good solutions in the research area, but drawbacks related these techniques include that: the structured-light patterns used are complicated, and need complex techniques to support; and complicated algorithms are required in the image processing, which results in a time-consuming processing and expensive to form an application.

It was observed from existing structured-light techniques that using only vertical or horizontal blackand-white strips is possible to provide enough deformation information of objects in the reconstruction processing. It is also feasible to use only one image for the object reconstruction to simplify the image capturing and processing. Following the 
observation, a simple method was proposed and implemented. A static mesh with black-and-white grids was designed to project onto objects in the proposed method. The image captured by a digital camera is used for the 3D shape reconstruction. The grid is composed of a single projection of $n \times n$ slits as shown in Figure 7. The dimension and distance of horizontal and vertical lines of the grid are predefined.

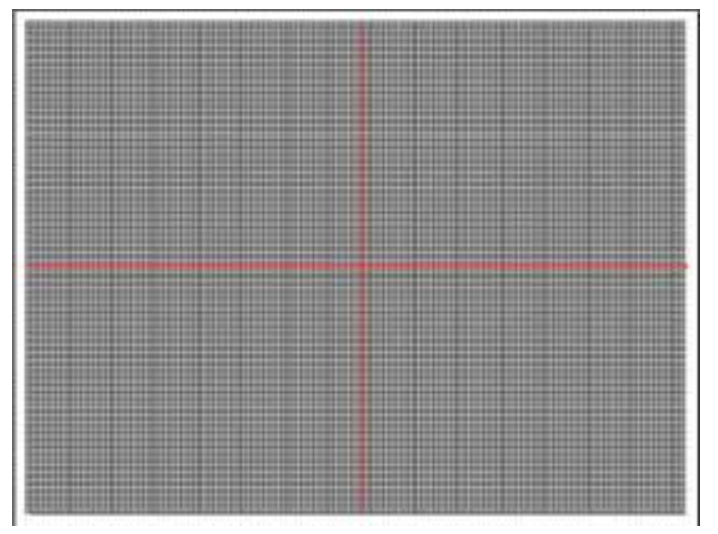

Figure 7. The projection grid used

The proposed method works as follows. Firstly, the camera and the projector are calibrated to obtain their intrinsic and extrinsic parameters, including focal length, the location of the image centre, the effective pixel size in the horizontal and vertical direction, and locations of camera, projector and object. A calibration pattern is used to link the image and a world coordinate system. The relation is established by detecting the same point of the calibration pattern in the image coordinate system and the world coordinate system. The camera then takes the scene where the grids are projected onto the object under reconstruction. The corners are detected and located in the world coordinate system. Finally, the depth of the object is recovered by the triangulation, and the mesh reconstruction forms whole shape of the object. A complete skeleton of the method is shown in Figure 8.

Differences of the proposed method from structured-light based methods are: a) the grid projected is static, it is not a set of grids projected in different time; b) the projected grid is black-and-white only; c) the thickness of the grid is considerably thin, only lines are projected and not strips or dots or columns with different color or grey intensity; d) only one picture is used to the shape acquisition.

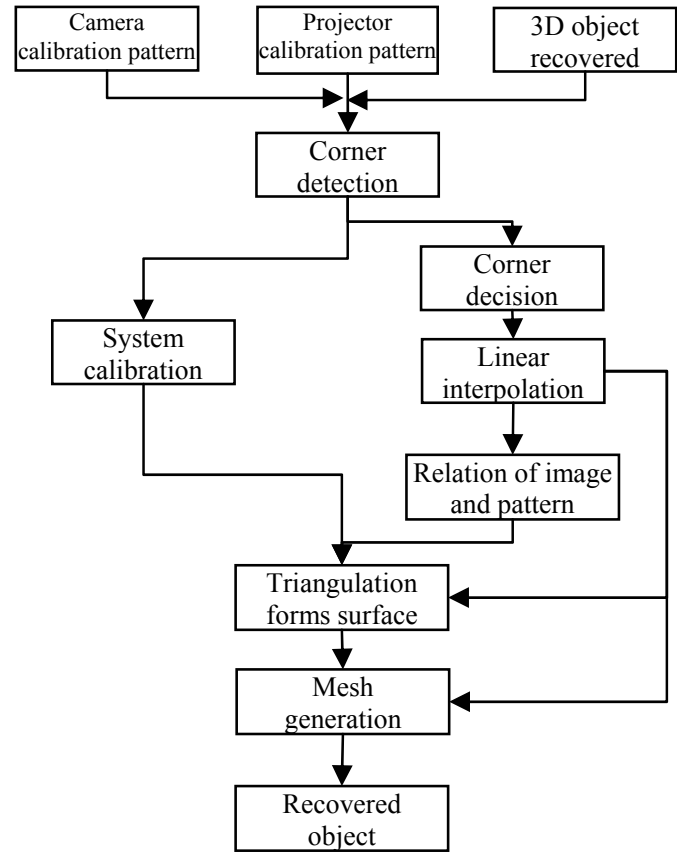

Figure 8. Skeleton of the proposed method.

\subsection{Techniques used in the proposed method}

The system calibration: A calibration pattern is used to find the relationship of camera and projector. The pattern is formed by equally spaced planar black squares on a white background simulating a chessboard. The calibration is performed only once if the location of camera and projector are not changed.

Corner detection: The corner detection finds grid locations of the calibration pattern and the object. Gonzales and Woods [18] defined corners as connected pixels that lie on the boundary between two regions. Corners are related to pixel intensity discontinuities in an image. Corner detection is typically a three-step process: noise smoothing, image enhancement and corner localization. Noise smoothing is the suppression of the image noise as much as possible. Image enhancement uses a special filter so that corners can be located as the local maxima in the filter's output. The corner detection used is based on Harris and Stephens' algorithm [19]. Then, linear interpolation is applied to increase the number of points detected by the corner detection. The image size used is $1600 \times 1200$ pixels. The centre of the image is located at $(800,600)$.

Height recovery using triangulation: The simplest case arises when optical axes of the camera and projector are parallel, and translation of the projection is only along $X$ axis. Let's consider the optical setting as shown in Figure 9. 


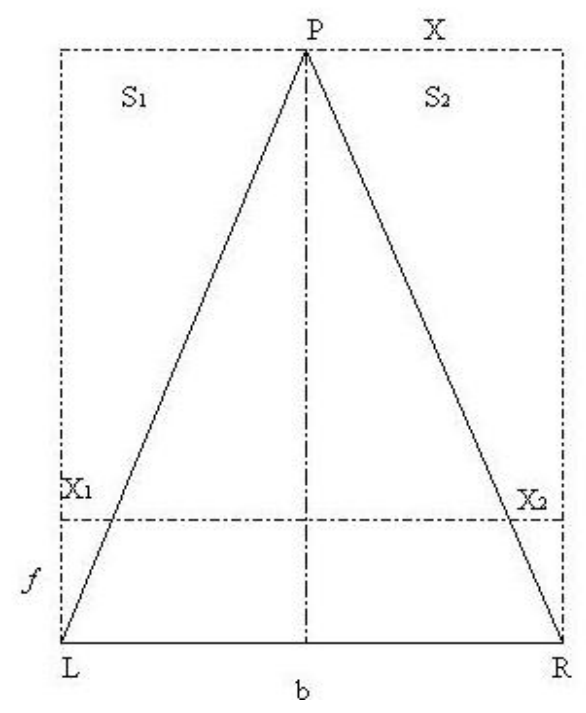

Z

Figure 9. Triangulation of parallel devises.

$L$ is a pinhole camera and $R$ is a projector with parallel optical axes referred to left and right respectively. $f$ is the focal length of both devices. The baseline is perpendicular to the optical axes. Let $b$ be the distance between two lens centers. $X Z$ is the plane where the optical axes lie, $X Y$ plane is parallel to the image plane of both devices, $X$ axis equals the baseline and origin $O$ of world reference system is the lens center of the left camera. $S_{1}$ and $S_{2}$ are projections of the base line. Triangles are identified as:

$\frac{X_{1}}{S_{1}}=\frac{f}{Z}=\frac{X_{2}}{S_{2}}$

$S_{2}=b-S_{1}$, therefore,

$\frac{X_{1}}{S_{1}}=\frac{X_{2}}{b-S_{1}}$

$b X_{1}=S_{1} X_{2}+S_{1} X_{1}=S_{1}\left(X_{1}+X_{2}\right)$

$S_{1}=\frac{b X_{1}}{X_{1}+X_{2}}$

$\mathrm{X}, \mathrm{Y}$ and $\mathrm{Z}$ are obtained as follows:

$$
\begin{aligned}
& Z=\frac{S_{1} f}{X_{1}}=\frac{f}{X_{1}} \cdot \frac{X_{1} b}{X_{1}+X_{2}}=\frac{b f}{X_{1}+X_{2}} \\
& X=\frac{X_{1} Z}{f}=b-\frac{X_{2} Z}{f} \\
& Y=\frac{Y_{1} Z}{f}
\end{aligned}
$$

When the devices are not parallel, the camera can be rotated with respect to the projector. The triangles relation can be established in the similar way. If there is the rotation angle $\theta, Z_{0}=\frac{b}{\tan (\theta)}$. Under the small angle approximation, it is still assumed that the right image plane is parallel to the left image plane. Rotation around $X$ axis $\phi$ only affects the $Y$ coordinate in reconstruction. Generally, given the translation vector $T$ and rotation matrix $R$ describing the transformation from camera to projector coordinates, the equation for stereo triangulation is:

$$
p^{\prime}=R T(p-T)
$$

Where $p$ and $p^{\prime}$ are the coordinates of $P$ in the left camera and right projector coordinates respectively, and $R T$ is the transpose matrix of $R$.

3D Mesh generation: The 3D mesh generation uses data cloud to form 3D shape of objects. The process is firstly to find all points that are in neighbourhoods. The number of valid neighbours is a parameter that can vary according to points considered. Four pixels are considered in neighbours surrounding each desired pixel. The next process is to smooth the generated mesh. For each point, the number of valid neighbours closed to the selected pixel in a certain distance is calculated. The final step is to generate triangles. Two triangles having the shortest diagonal are created from four pixels in neighbourhoods only if the points are considered close to each other.

\subsection{Examples and analysis}

Two objects were used as samples to test the performance of the proposed method. The evaluation was based on the comparison of the accuracy obtained by the proposed method and measured by the 3D laser scanner discussed in Section 3. The selected objects are an electric kettle and a foot model. The image resolution is $1200 \times 1600$ pixels. The electric kettle image, projected grid on the object and the recovered shape are shown in Figure 10. The detected corners are shown in Figure 10 (c). A linear interpolation is applied to increase the number of points. The final shape is recovered by the triangulation as shown in Figure 10 (d). The other object is a foot model. Figure 11 shows the object acquisition, corner detection and the reconstructed foot by the proposed method.

In order to evaluate the developed method, the recovered shape was compared with shape recovered by the 3D laser scanner. The laser scanner is 

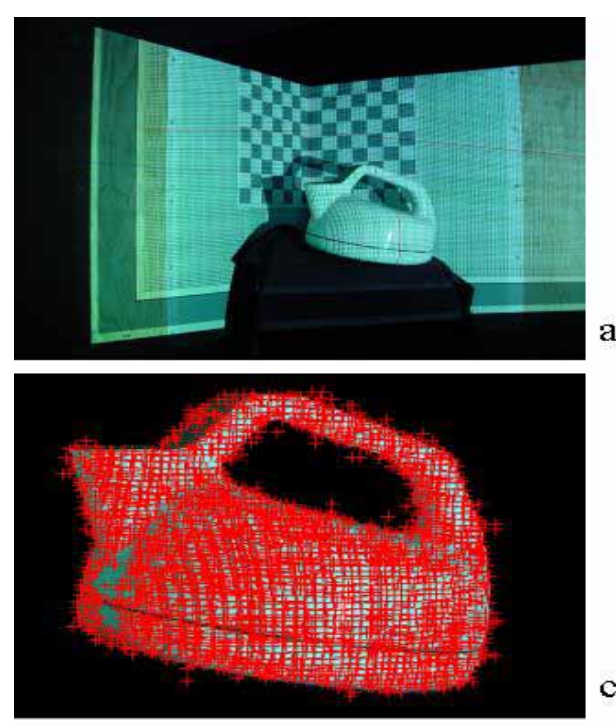

b

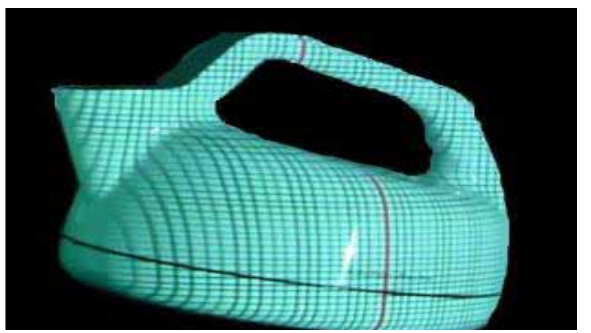

d

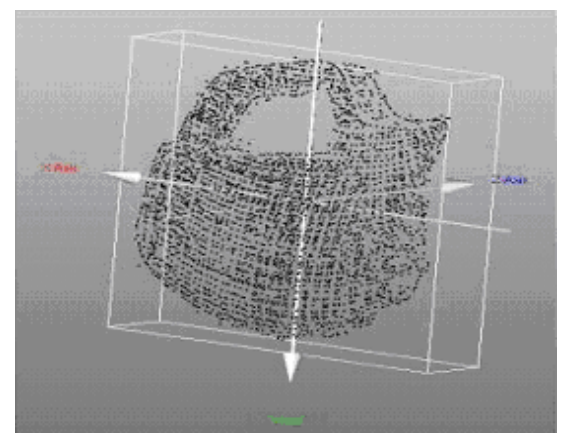

Figure 10. The electric kettle: a) Image to be reconstructed with the projected grid, b) the projected grid, c) corner detection, d) recovered shape.
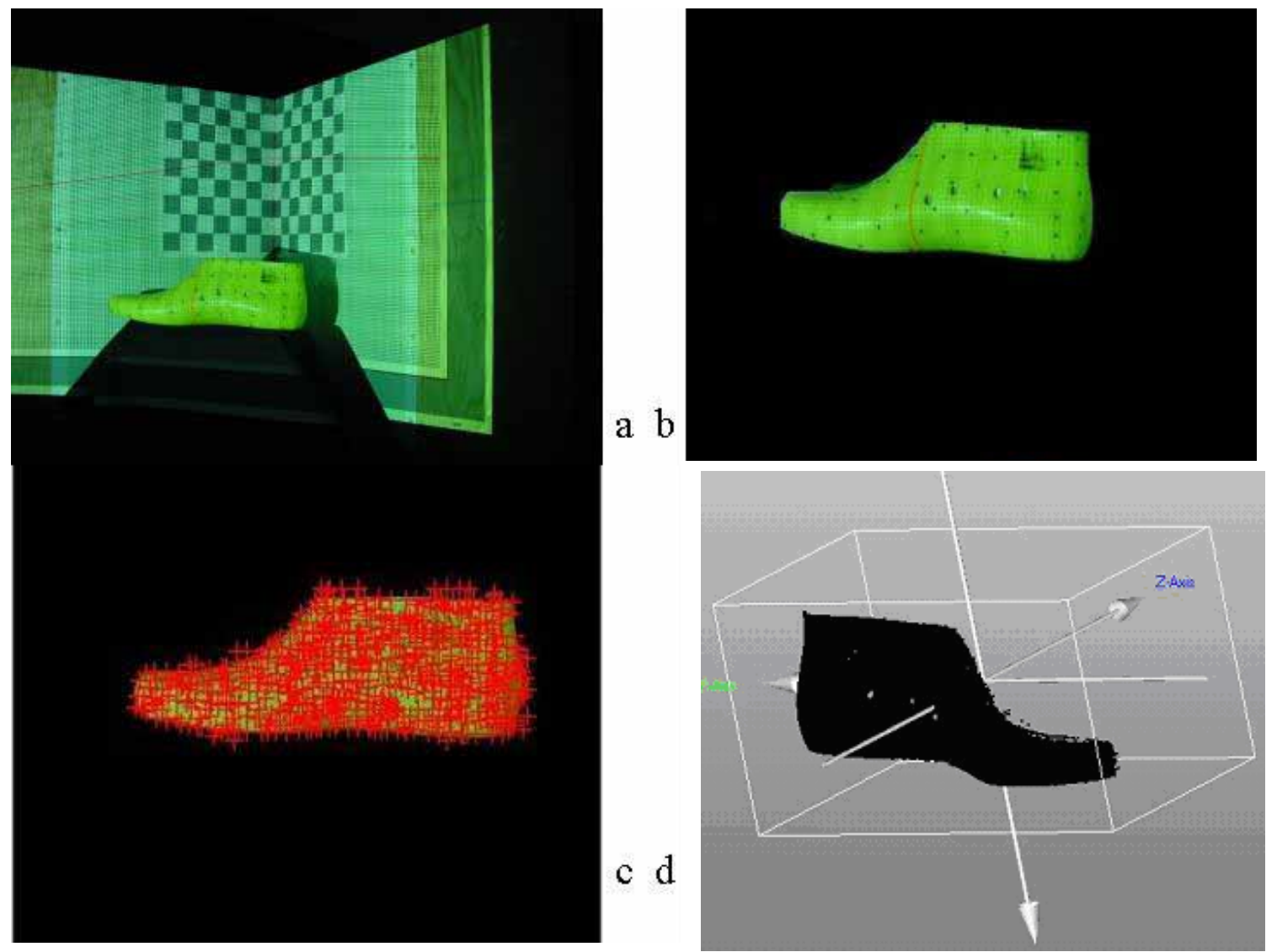

Figure 11. The foot model: a) object with the projected grid, b) the projected grid, c) corner detection d) recovered foot shape. 
considered as an accurate tool to get real values of the objects. X, Y, Z are directions of width, height and depth of the objects respectively. The error was calculated by the difference between values obtained by the scanner and the value recovered by proposed method. The maximum errors are 1.24, 1.90 and 1.91 $\mathrm{mm}$ in $\mathrm{X}, \mathrm{Y}$, and $\mathrm{Z}$ directions respectively for the kettle, which gives $5.9 \% 5.5 \%$ and $2.2 \%$ relative errors respectively. The maximum errors are 1.41, 1.47, and $2.86 \mathrm{~mm}$ in $\mathrm{X}, \mathrm{Y}$, and $\mathrm{Z}$ directions, respectively for the foot model, which gives $4.6 \% 5.4 \%$ and $3.7 \%$ relative errors respectively.

From the accuracy comparison, the proposed approach generated a close approximation to the real objects. It can be observed that the curvature along the body of the kettle is similar to the original shape. It is also identified that the shape is close to the real body, handle, and spout. The slot between walls of the spout and handle are also presented in the reconstructed shape. The length and height recovered are almost the same. The recovered results of the foot model have the similar results as the kettle. The toe of the foot has curvature because the position of the image captured. The picture taken is not able to see the complete lines projected. Better orientation of the object to acquire image could generate a better result.

These examples demonstrated the performance of the proposed method. The method generates a good approximation of the objects. Some difficulties are presented when reconstructing depth, especially if the surface is sharply curved. The major problem is shown in the sharp edges. The projected lines are lost or become smaller in curve areas, making the reconstruction difficulty. Smooth curvatures are well reconstructed, as the case of the electric kettle. Linear objects are well recognized and reconstructed as the case of the foot. Problems related to accuracy in the length and height could be fixed by focusing on borders of the acquired image. In the case of concave objects or surfaces with holes, the algorithm will not work. The reason is that the projected lines are not present in the holes, making the reconstruction process impossible.

\section{Conclusions and further work}

This paper discussed the 3D shape recovery and techniques in product reverse design. Generally, noncontact device is a convenient technology for 3D data acquisition. The time used for data acquisition is shorter than other methods. There are different types of 3D scanners available based on methods of capturing objects. These scanners provide different measuring range and accuracy. Users have to correctly select a suitable 3D scanning method for their particular needs.
This paper proposed a simple method to recover 3D shape of objects using the structured-light and triangulation based on the analysis of current scanning technology. A grid is projected onto the surface of an object to improve recovery accuracy and simplify the image processing. The deformed grids on the image are used to find the 3D position of each recovered point of the object by triangulation. The calibration for the camera and projector creates the link between the object and the system. The measuring accuracy was evaluated.

The accuracy of the proposed method can be improved by increasing the number of points processed, which can be achieved using a camera with higher resolution. Problems related to precision could also be improved by focusing on borders of the image. Besides corner detection, edge detection could be performed to find complete lines across the object. Curvatures also present a problem that must be improved. A different position of the camera or the projector could be a solution of this problem. Another possibility to improve the accuracy is to increase the intensity of grid and precision of projected lines onto the object.

\section{Acknowledgement}

This research is supported by Canadian Foundation for Innovation (CFI) and Canadian NSERC Discovery Grants. Thanks to Mr. Jakob Blosser for his work in the scanner test.

\section{References}

[1] L. Li, N. Schemenauer, X. Peng, Y. Zeng and P. Gu, "A reverse engineering system for rapid manufacturing of complex objects", Robotics and Computer-Integrated Manufacturing, 2002, Vol. 18, No. 1, pp.53-67.

[2] X. Yan, K. Yamazaki and J. Liu, "Reverse engineering of machining operation planning", International Journal of Production Research, 2001, Vol.39, No.9, pp.18371849.

[3] Y. ZHANG, "Research into the engineering application of reverse engineering technology", Journal of Materials Processing Technology, 2003, Vol.139, No. 13, pp.472-475.

[4] L. M. Galantucci, G. Percoco and R. Spina, "Evaluation of rapid prototypes obtained from reverse engineering", Proceedings of the Institution of Mechanical Engineers, Part B: Journal of Engineering Manufacture, 2003, Vol.217, No.11, pp.1543-1552.

[5] C-X J. Feng, "Internet-based reverse engineering", International Journal of Advanced Manufacturing Technology, 2003, Vol.21, No.2, pp.138-144.

[6] J. Yan and S. L. De, "Reverse Engineering of Sheet Metal Parts Using Machine Vision”, Proceedings of the 
ASME Design Engineering Technical Conference, 2003, 1B, pp.1085-1095.

[7] X. Li, Z. Li, H. Tian and Z. Ziping, "A study of a reverse engineering system based on vision sensor for free-form surfaces", Computers and Industrial Engineering, 2001, Vol.40, No.3, pp.215-227.

[8] K-Y, Bae and B. Benhabib, "A hybrid scheme incorporating stereo-matching and shape-from-shading for spatial object recognition", Proceedings of the Institution of Mechanical Engineers, Part B: Journal of Engineering Manufacture, 2003, Vol.217, No.11, pp. $1533-1542$

[9] F.A.R. Martins, J.G. Garcia-Bermejo, E. Zalama and J.R. Peran, "An optimized strategy for automatic optical scanning of objects in reverse engineering", Proceedings of the Institution of Mechanical Engineers, Part B: Journal of Engineering Manufacture, 2003. Vol. 217, No.8, pp.1167-1171.

[10] K.H. Lee, H. Park and S. Son, "A framework for laser scan planning of freeform surfaces", International Journal of Advanced Manufacturing Technology, 2001. Vol.17, No.3, pp.171-180.

[11] B. Lu, Z. Jiang and Y. Luo, "Measurement of threedimensional profiles with multi structure linear lighting”, Robotics and Computer-Integrated Manufacturing, 2003. Vol.19, No.6, pp.493-499.

[12] S. Son, S. Kim and K. Lee, "Path planning of multipatched freeform surfaces for laser scanning", International Journal of Advanced Manufacturing Technology, 2003, Vol.22, No.5-6, pp.424-435.

[13] A. Akgunduz, D. Zetu and P. Banerjee, "Viewpointbased shape recovery from multiple views", Artificial Intelligence for Engineering Design, Analysis and Manufacturing: AIEDAM, 2000, Vol.14, No.5, pp.359372.

[14] V.H. Chan, C. Bradley and G.W. Vickers, "Multi-sensor approach to automating co-ordinate measuring machinebased reverse engineering", Computers in Industry, 2001, Vol. 44, No. 2, Vol. 105-115.

[15] E. M. Petriu, T. Bieseman, N. Trif, W.S. McMath and K. Yeung, "Visual object recognition using pseudo-random grid coding", Proceedings of the IEEE/RSJ International Conference on Intelligent Robots and Systems, 1992, 1617-1624.

[16] O. Hall-Holt and S. Rusinkiewicz, "Stripe Boundary codes for real time structures light range scanning of moving objects". Stanford computer graphics labs, Stanford University, 2003.

[17] T. Miyasaka, K. Kuroda, M. Hirose and K. Araki, "High speed 3-D measurement system using incoherent light source for human performance analysis", Proceedings of the 19th Congress of The International Society for Photogrammetry and Remote Sensing, 2000, pp.65-69.

[18] R.C. Gonazalez and R.E. Woods, Digital Image Processing, Second edition, Prentice Hall, 2002.

[19] C.G. Harris and M.J. Stephens, "A combined corner and edge detector". Proceedings Fourth Alvey Vision Conference, Manchester. 1988, pp.147-151. 